\title{
ESTIMATION OF ASSORTATIVE MATING PREFERENCES IN THE ARCTIC SKUA
}

\author{
J.W. F. DAVIS and P. O'DONALD \\ Department of Genetics, University of Cambridge, Milton Road, \\ Cambridge CB4 1 XH, England
}

Received 20.x.75

\begin{abstract}
SUMMARY
Methods are described for the maximum likelihood estimation of mating preferences in models of assortative mating for monogamous and polygamous organisms. These methods are applied to data of matings of the three phenotypes, pale, intermediate and dark of the Arctic Skua. The data were obtained by exhaustive surveys of the Arctic Skua populations on the islands of Fair Isle and Foula. The data give evidence of significant assortative mating of pale birds on Foula and intermediate birds on Fair Isle. The combined data show that there is very highly significant assortative mating, but only of intermediates. In previous surveys, data, in which intermediates and darks were not distinguished, were obtained from a number of islands in the Shetlands. These data, combined with the present data, show that the overall assortative mating of pale is very highly significant with no evidence of heterogeneity. The assortative mating of intermediate birds on Fair Isle agrees with other evidence showing that intermediate males have an advantage as a result of sexual selection.
\end{abstract}

\section{INTRODUCTION}

MODELs of assortative mating (O'Donald, 1960; Karlin, 1969) can be described in terms of preferences among some individuals to mate with others phenotypically similar or dissimilar to themselves. The proportions of individuals who mate preferentially can be estimated from the actual numbers of the different matings. O'Donald, Davis and Broad (1974) obtained such estimates from matings of the phenotypes of the Arctic Skua (Stercorarius parasiticus), a monogamous seabird that lives in loose colonies. O'Donald (1960) analysed models of assortative mating for monogamous species and Karlin (1969) the corresponding models for polygamous species. The models, although simple, are biologically quite realistic. As many experiments have shown, the females often have mating preferences for particular male characteristics: when given a choice, they mate more often with males having a particular phenotype. The mechanisms by which the females determine their preferences are unknown, but presumably depend on thresholds at which their mating responses are released by the different male phenotypes. Male competition may also produce differences in the chances of mating. Whatever particular behavioural mechanisms determine the different chances of mating the term "mating preference" is used to describe the observed effects. Male preference has been observed less often. It has been shown to occur in some species of butterflies (Burns, 1966). We describe the models in terms of female preferences, but they would be valid if males alone, or both sexes, had mating preferences.

If the assortative mating affects characteristics whose genetic determina- 
tion is known, the models can then be used to predict the evolutionary consequences of the mating preferences (O'Donald, 1960; Karlin, 1969). In this paper, however, we consider the problem of estimating mating preferences that would explain observations of assortative mating.

\section{Models of assortative mating}

In the models with monogamy, we assume that the preferential matings take place first: unmated individuals then mate together at random. The Arctic Skua has three main phenotypes: pale, intermediate and dark. The intermediates show a wide range of variation and may be difficult to distinguish in the field from the darks. Since pale and dark birds are homozygous and intermediates heterozygous, the models must allow for assortative mating within each phenotype: changes in the frequencies of the genotypes can then be predicted. If the three phenotypes are called $A, B$ and $C$, with frequencies $u, v$ and $w$ and probabilities $\alpha, \beta$ and $\gamma$ of mating assortatively, then a proportion of the population $1-R=\alpha u+\beta v+\gamma w$ mates preferentially and the proportion $R$ mates at random. The random matings take place with $A$, $B$ and $C$ at frequencies $u(1-\alpha) / R, v(1-\beta) / R$ and $w(1-\gamma) / R$. The preferential matings, $A \times A, B \times B$ and $C \times C$, take place with frequencies $\alpha u, \beta v$ and $\gamma w$. In the models with polygamy, the males who mated preferentially are still available to mate at random: the random matings thus take place with $A, B$ and $C$ at frequencies $u, v$ and $w$ in males and $u(1-\alpha) / R, v(1-\beta) / R$ and $w(1-\gamma) / R$ in females. Table 1 shows the frequencies of the matings according to these models.

TABLE 1

Frequencies of matings when phenotypes A, B, and $\mathrm{C}$ mate assortatively with probabilities $\alpha, \beta$ and $\gamma$.

\begin{tabular}{clc} 
Matings & $\overbrace{\text { With monogamy }}$ Frequency of matings & With polygamy \\
$A \times A$ & $\alpha u+u^{2}(1-\alpha)^{2} / R$ & $\alpha u+u^{2}(1-\alpha)$ \\
$A \times B$ & $2 u v(1-\alpha)(1-\beta) / R$ & $u v(2-\alpha-\beta)$ \\
$A \times C$ & $2 u w(1-\alpha)(1-\gamma) / R$ & $u w(2-\alpha-\gamma)$ \\
$B \times B$ & $\beta v+v^{2}(1-\beta)^{2} / R$ & $\beta v+v^{2}(1-\beta)$ \\
$B \times C$ & $2 v w(1-\beta)(1-\gamma) / R$ & $v w(2-\beta-\gamma)$ \\
$C \times C$ & $\gamma w+w^{2}(1-\gamma)^{2} / R$ & $\gamma w+w^{2}(1-\gamma)$ \\
\multicolumn{3}{c}{$R=1-\alpha u-\beta v-\gamma w$}
\end{tabular}

We have been unable to find explicit solutions to the equations for maximum likelihood when all independent parameters are to be fitted to the data. The maximum likelihood can be found by trial and error by introducing random perturbations into arbitrary values of the parameters until higher values of the log likelihoods are found. By making the random perturbations smaller and smaller, the values of log likelihood can be made to converge on the maximum. This method was used by O'Donald, Davis and Broad to fit two parameters of the mating preferences to data on numbers of matings of the three phenotypes of the Arctic Skua.

When only one parameter of the mating preference is to be fitted, explicit solutions can be obtained for the values at maximum likelihood. If 
$\beta=\gamma=0, \alpha$ and $u$ are the only parameters to be estimated, the phenotypes $B$ and $C$ being lumped together as $B$ with frequency $1-u$. If $\alpha=\beta=\gamma$, the models for monogamy and polygamy become identical: $\alpha$ and $u$ are again the only parameters to be estimated. Table 2 shows the frequencies of the matings according to these models.

\section{TABLE 2}

Frequencies of matings with one parameter of assortative mating

\begin{tabular}{|c|c|c|c|c|}
\hline \multirow[b]{3}{*}{ Matings } & \multirow{3}{*}{$\begin{array}{l}\text { Observed } \\
\text { numbers } \\
\text { of matings }\end{array}$} & \multicolumn{3}{|c|}{ Frequencies of matings } \\
\hline & & \multicolumn{2}{|c|}{ When $\beta=\gamma=0$} & \multirow{2}{*}{$\begin{array}{c}\text { When } \alpha=\beta=\gamma \\
\text { (Model 3) }\end{array}$} \\
\hline & & Monogamy (Model 1) & Polygamy (Model 2) & \\
\hline$A \times A$ & a & $\alpha u+u^{2}(1-\alpha)^{2} /(1-\alpha u)$ & $\alpha u+u^{2}(1-\alpha)$ & $\alpha u+u^{2}(1-\alpha)$ \\
\hline$A \times B$ & $\mathrm{~b}$ & $2 u(1-u)(1-\alpha) /(1-\alpha u$ & $u(1-u)(2-\alpha)$ & $2 u(1-u)(1-\alpha)$ \\
\hline$B \times B$ & c & $(1-u)^{2} /(1-\alpha u)$ & $(1-u)^{2}$ & $\alpha(1-u)+(1-u)^{2}(1-\alpha)$ \\
\hline
\end{tabular}

\section{Maximum likelihood estimation}

Solutions have been obtained to the equations for maximum likelihood for the three models given in table 2. In Model 1, matings are monogamous, with $\beta=\gamma=0$. The $\log$ likelihood is given by

$\log L=a \cdot \log (\alpha+u-2 \alpha u)+b \cdot \log (1-\alpha)+(a+b) \cdot \log u$

$$
+(b+2 c) \cdot \log (1-u)-n \cdot \log (1-\alpha u) \text {. }
$$

Thus at a maximum when $\frac{\partial \log L}{\partial u}=0$ and $\frac{\partial \log L}{\partial \alpha}=0$,

we have the estimates $\hat{u}$ and $\hat{\alpha}$ where

$$
\hat{u}=\left(a+\frac{1}{2} b\right) / n
$$

and $\hat{\alpha}$ is found as the solution of the equation

$$
\begin{aligned}
\alpha^{2}[c u(2 u-1)]-\alpha\left[u^{2}(a+2 b+3 c)-u(2 a+3 b+c)+a+b\right] & \\
& +n u^{2}-u(2 a+b)+a=0 .
\end{aligned}
$$

The information matrix is defined by the equations

where

$$
\begin{aligned}
& I_{11}=-\mathscr{E} \frac{\partial^{2} \log L}{\partial u^{2}} \\
& I_{12}=-\mathscr{E} \frac{\partial^{2} \log L}{\partial \alpha \partial u} \\
& I_{22}=-\mathscr{E} \frac{\partial^{2} \log L}{\partial \alpha^{2}}
\end{aligned}
$$

$$
I_{11}=\frac{n u(1-2 \alpha)^{2}}{(1-\alpha u)(\alpha+u-2 \alpha u)}+\frac{n(2-u-\alpha)}{u(1-\alpha u)}+\frac{2 n}{1-u}-\frac{n \alpha^{2}}{(1-\alpha u)^{2}}
$$




$$
\begin{gathered}
I_{12}=\frac{n u}{(1-\alpha u)(\alpha+u-2 \alpha u)}-\frac{n}{(1-\alpha u)^{2}} \\
I_{22}=\frac{n u(1-2 u)^{2}}{(1-\alpha u)(\alpha+u-2 \alpha u)}+\frac{2 n u(1-u)}{(1-\alpha)(1-\alpha u)}-\frac{n u^{2}}{(1-\alpha u)^{2}}
\end{gathered}
$$

From these values we find the variances and covariances of the estimates are given by

$$
\begin{aligned}
\operatorname{var}(\hat{u}) & =I_{22} /\left(I_{11} I_{22}-I_{12}^{2}\right) \\
\operatorname{var}(\hat{\alpha}) & =I_{11} /\left(I_{11} I_{22}-I_{12}^{2}\right) \\
\operatorname{cov}(\hat{u}, \hat{\alpha}) & =I_{12} /\left(I_{11} I_{22}-I_{12}^{2}\right)
\end{aligned}
$$

In Model 2, matings are polygamous with $\beta=\gamma=0$. We have the equation

$\log L=a \cdot \log (\alpha+u-\alpha u)+b \cdot \log (2-\alpha)+(a+b) \cdot \log u+(b+2 c) \cdot \log (1-u)$ giving maximum likelihood estimates

and

$$
\begin{aligned}
& \hat{u}=1-\sqrt{1-(a+b) / n} \\
& \hat{\alpha}=\frac{2 a(1-u)-b u}{(a+b)(1-u)}
\end{aligned}
$$

$$
\begin{aligned}
& I_{11}=\frac{n u(1-\alpha)^{2}}{(\alpha+u-\alpha u)}+\frac{n(2-u)}{u}+\frac{n(2-\alpha u)}{(1-u)} \\
& I_{12}=\frac{n u}{(\alpha+u-\alpha u)} \\
& I_{22}=\frac{n u(1-u)^{2}}{(\alpha+u-\alpha u)}+\frac{n u(1-u)}{(2-\alpha)}
\end{aligned}
$$

In this model the estimate of $u$ is not simply the proportion of the $A$ phenotypes because some males mate more than once and their frequencies in the matings differ from their frequencies as individuals.

In Model 3, with $\alpha=\beta=\gamma$, we have

$\log L=a \cdot \log (\alpha+u-\alpha u)+c \cdot \log (1-u+\alpha u)+b \cdot \log (1-\alpha)$

$$
+(a+b) \cdot \log u+(b+c) \cdot \log (1-u) \text {. }
$$

The maximum likelihood estimates are then found to be

$$
\begin{aligned}
& \hat{u}=\left(a+\frac{1}{2} b\right) / n \\
& \hat{\alpha}=\frac{a c-\frac{1}{4} b^{2}}{\left(a+\frac{1}{2} b\right)\left(c+\frac{1}{2} b\right)}
\end{aligned}
$$

This estimate for $\alpha$ represents a simple measure of departure from random mating which is the expected result for this symmetric model. Then 


$$
\begin{aligned}
& I_{11}=\frac{n u(1-\alpha)^{2}}{\alpha+u-\alpha u}+\frac{n u(1-u)(1-\alpha)^{2}}{1-u+\alpha u}+\frac{n(2-\alpha-u+\alpha u)}{u}+\frac{n(1+u-\alpha u)}{(1-u)} \\
& I_{12}=\frac{n u}{(\alpha+u-\alpha u)}-\frac{n(1-u)}{(1-u+\alpha u)} \\
& I_{22}=\frac{n u(1-u)^{2}}{(\alpha+u-\alpha u)}+\frac{n u^{2}(1-u)}{(1-u+\alpha u)}+\frac{2 n u(1-u)}{(1-\alpha)}
\end{aligned}
$$

\section{Assortative mating in the Arctic Skua}

Data on assortative mating in the Arctic Skua were first obtained for matings of pale and non-pale phenotypes (O'Donald, 1960). These data were collected by a number of observers. The non-pale birds were not classified into intermediate and dark phenotypes: these phenotypes are more difficult to distinguish from each other than from the sharply distinct pales. We have since classified the birds into four phases: pale, intermediate, darkintermediate and dark. Intermediates and dark-intermediates represent a continuous range of variation and genetic data suggest they are nearly all heterozygotes. In new data which are given in this paper, intermediates and dark-intermediates have been classified together as intermediates. There may be mating preferences for all three phenotypes which can be estimated from data on numbers of matings between the different phenotypes.

O'Donald, Davis and Broad (1974) gave preliminary data on numbers of matings of all three phenotypes based on a survey carried out in 1974 on two islands in the Shetlands which have large breeding populations of Arctic Skuas. These are the islands of Fair Isle and Foula. The Arctic Skuas on Fair Isle are being studied in great detail and all pairs are known and the birds ringed. In 1974 a brief survey of the large population of Arctic Skuas on Foula was carried out by quartering the breeding grounds and observing pairs of birds giving distraction displays. The analysis of these data showed that the pale phenotypes mated assortatively on Foula and intermediate phenotypes mated assortatively on Fair Isle. In 1975, in addition to carrying out our detailed study on Fair Isle, we also studied the Foula population intensively, trying to find and mark every nest on the island. We were mainly concerned to ring the adults and chicks with colour-coded rings to analyse migration and survival rates, but at the same time we obtained the data on the phenotypes of the breeding pairs. We were able to classify the phenotypes of 256 pairs of nesting birds out of the total of 280 nests we found. The data for 1975 are shown in table 3 . The overall deviation from random mating is highly significant in the combined data. However the values of $\chi^{2}$ are smaller than the corresponding values for the data of 1974 . In particular there was a highly significant deviation from random mating in the data from Foula for 1974 but not for 1975. This difference has arisen because of the smaller proportion of pale $\times$ pale matings observed on Foula in 1975. These matings give rise to the highly significant assortative mating of pale phenotypes in the data for 1974. Possibly the high frequency of pale $\times$ pale matings on Foula in 1974 was a freak result: a number of these matings broke up in the following year. There was certainly a reduction in the number of pale $\times$ pale matings observed in some areas of the colony in 
1975 in spite of the fact that the colony was surveyed much more thoroughly by the finding of each nest. This gave data on about 50 per cent more pairs. On Foula the Arctic Skuas breed in three main areas: one around the airstrip in the south, which is the densest part of the population; one among peat cuttings in the middle of the crofting area; and one in the north of the island. There is no heterogeneity in the frequencies of the matings, however, and the numbers in table 3 are those for the island as a whole. On Fair Isle, the Arctic Skuas live in one large colony, with only a few pairs separated from the rest.

Table 4 gives a detailed analysis of the data from Fair Isle and Foula in 1975. The parameters of assortative mating, $\alpha$ for pale, $\beta$ for intermediate and $\gamma$ for dark, are fitted to the data for each island separately and the lumped totals for both islands. Table 4 shows a curious result. Fitting the parameter $\beta$ to the data from Fair Isle leaves no significant residual deviation

\section{Table 3}

Observed numbers of matings of Arctic Skuas on Fair Isle and Foula in 1975

\begin{tabular}{lccc}
\multicolumn{1}{c}{ Matings } & \multicolumn{2}{c}{ Numbers of matings } \\
\cline { 2 - 2 } & Fair Isle & Foula & Totals \\
Pale $\times$ pale & 7 & 26 & 33 \\
Pale $\times$ intermediate & 25 & 58 & 83 \\
Pale $\times$ dark & 15 & 28 & 43 \\
Intermediate $\times$ intermediate & 56 & 70 & 126 \\
Intermediate $\times$ dark & 23 & 58 & 81 \\
Dark $\times$ dark & 7 & 16 & 23 \\
$\quad$ Totals & 133 & 256 & 389 \\
Chi-squared for deviation from random mating & $8 \cdot 219$ & $6 \cdot 160$ & 11.547
\end{tabular}

from the theoretical model. The single degree of freedom for $\beta$ corresponds to a highly significant value of $\chi^{2}$. The same is true when the parameter $\alpha$ is fitted to the data from Foula. The data for 1975 give results very similar to those for 1974 except for the higher estimated value of $\alpha$ for 1974 representing the higher proportion of pale $\times$ pale matings in 1974. Yet when the data are combined, the heterogeneity between islands is close to expectation. The residual heterogeneity when all three parameters of the mating system, $\alpha, \beta$ and $\gamma$, are fitted to the combined data is given by $\chi^{2}=4.645$ for 3 degrees of freedom which has the probability $\mathrm{P} \bumpeq 0 \cdot 2$. The parameter $\beta$ alone is quite sufficient to fit the combined data and there is no heterogeneity between islands, for $\chi^{2}=6.609$ with 5 degrees of freedom which has probability in the range $0 \cdot 3>P>0 \cdot 2$. This analysis suggests that significant assortative mating takes place only among intermediates and this is not heterogeneous between islands.

In spite of this conclusion the data from Foula give evidence of highly significant assortative mating of pale. The data can be analysed using the formulae given in section 3 . The intermediates and darks must be lumped together into the single class of non-pales. The formulae can be used similarly for the analysis of assortative mating of intermediates in Fair Isle, pales and darks being lumped together as non-intermediate. The previous data (O'Donald, 1960) can also be included in the analysis of pale and nonpale matings. These data were collected at a number of different colonies 





of Arctic Skuas in Shetland by several observers including O'Donald. They show very significant assortative mating of pale and non-pale phenotypes with no heterogeneity between colonies. Table 5 gives the data for Fair Isle and Foula in 1975 together with the total numbers of matings observed in different colonies during the earlier work. The detailed data can be found in O'Donald (1960). Values of $\alpha$ have been estimated for model 1, when $\beta=\gamma=0$, and for the symetric model, model 3 , when $\alpha=\beta=\gamma$. These estimates are shown in table 5 . The overall assortative mating between pales and non-pales is very highly significant, particularly in the earlier data. The values of $\chi^{2}$ given in the table are those for the overall deviation from random mating. They are similar to values of $\chi^{2}$ obtained for the test of

Table 5

Assortative mating of pale and non-pale phenotypes

\begin{tabular}{|c|c|c|c|c|}
\hline Mating & Fair Isle 1975 & Foula 1975 & $\begin{array}{c}\text { Data from } \\
\text { O'Donald (1960) }\end{array}$ & Totals \\
\hline Pale $\times$ pale & 7 & 26 & 38 & 71 \\
\hline Pale $\times$ non-pale & 40 & 86 & 120 & 246 \\
\hline Non-pale $\times$ non-pale & 86 & 144 & 218 & 448 \\
\hline$\chi^{2}$ & $0 \cdot 663$ & $5 \cdot 522$ & $11 \cdot 114$ & $17 \cdot 343$ \\
\hline Model $1\left\{\begin{array}{l}\hat{\alpha} \\
\operatorname{var}(\hat{\alpha})\end{array}\right.$ & $\begin{array}{l}0 \cdot 0870 \\
0 \cdot 01253\end{array}$ & $\begin{array}{l}0 \cdot 1907 \\
0.006621\end{array}$ & $\begin{array}{l}0.2192 \\
0.004393\end{array}$ & $\begin{array}{l}0 \cdot 1919 \\
0.002197\end{array}$ \\
\hline Model $3\left\{\begin{array}{l}\hat{\alpha} \\
\operatorname{var}(\hat{\alpha})\end{array}\right.$ & $\begin{array}{l}0 \cdot 0706 \\
0 \cdot 008519\end{array}$ & $\begin{array}{l}0 \cdot 1469 \\
0 \cdot 004311\end{array}$ & $\begin{array}{l}0 \cdot 1719 \\
0 \cdot 002993\end{array}$ & $\begin{array}{l}0 \cdot 1506 \\
0 \cdot 001476\end{array}$ \\
\hline
\end{tabular}

significance of the deviation of the estimate $\hat{\alpha}$ from its expected value $\alpha=0$ under random mating, and also similar to the values by which the overall $\chi^{2}$ is reduced when the parameter $\alpha$ is fitted to the data of Fair Isle and Foula 1975 as shown in table 4 . The differences in these values of $\chi^{2}$ are essentially caused by differences in the parameterisation of the specific model implied by the test of significance.

In spite of the differences in the estimates for $\alpha$ for the three sets of data in table 5, there is no heterogeneity between the estimates. If the estimates for the combined data are used to fit each set of data for the calculation of $\chi^{2}$, we get for model 1 the value for heterogeneity of

$$
\chi^{2}=0 \cdot 1726
$$

and for model 3 of

$$
\chi^{2}=0 \cdot 1547
$$

These values of $\chi^{2}$ have 2 degrees of freedom and indicate close agreement with expectation. The data show that there is highly significant assortative mating of pales and non-pales, with no detectable heterogeneity between colonies of birds.

These analyses show that significant assortative mating for pale and intermediate phenotypes takes place in Arctic Skua populations. The analysis of the data shows that pales mate assortatively on Foula and intermediates on Fair Isle: the parameter of assortative mating for pale accounts for almost the whole of the deviation from random mating on Foula; the parameter of assortative mating for intermediate does so for Fair Isle. And yet the analysis gives no evidence of heterogeneity. The populations of Skuas in 1975 could 
be considered to be random samples from a population in which both pales and intermediates mate assortatively. Tests of heterogeneity are very insensitive, particularly when some of the phenotypes are uncommon so that matings between them are rare. Small differences in the numbers of matings also introduce large differences in the estimates of the parameters. This is partly responsible for the differences between the estimates of assortative mating for pale in 1974 and 1975 on Foula. The slight reduction in pale $\times$ pale matings in some areas together with a considerable increase in data obtained by the more extensive survey of the population has thus rather drastically reduced the estimate of the assortative mating preference for pale.

\section{Discussion: sexual selection and assortative mating}

O'Donald (1972) and O'Donald, Wedd and Davis (1974) showed that sexual selection favoured the intermediate and dark phenotypes of the Arctic Skua. Intermediate and dark males who are mating with a particular female for the first time, breed at an earlier average date than pale males. There are no differences in the average breeding dates of the phenotypes among females or among males mating with the same female in later years. The darker males have a higher probability of finding a mate earlier in the breeding season. This gives them a selective advantage because earlier pairs fledge more offspring on average- - a fairly common phenomenon in birds. Assuming that the females have preferences to mate with the darker males, models can be set up giving the probability that a male of a particular phenotype will find a mate at some period of time during the breeding season. O'Donald (1973, 1974) and O'Donald, Wedd and Davis (1974) described some specific models of mating preferences. They fitted these models to the observed distributions of the breeding times of the males by finding estimates of the mating preferences that produced theoretical distributions of breeding times with maximum likelihood.

No assumptions were made in these models of female mating preferences about the females' phenotypes. The parameters of the mating preferences were simply the proportions of all females who would mate with particular phenotypes of males if they could. Sexual selection takes place in the models because the favoured males gain mates earlier in the breeding season and hence gain a selective advantage from their increased reproductive success. O'Donald, Wedd and Davis (1974) calculated the selective advantage from the empirical relationship between breeding time and reproductive success. This is exactly how Darwin (1871) originally considered sexual selection might operate in a monogamous species. If all the males in a monogamous species eventually find mates and their reproductive success does not vary with breeding time, then no selection will take place, whatever mating preferences some males may have in their favour.

In positive assortative mating it is assumed that mating preferences for a particular phenotype are restricted to individuals of that phenotype. In the simple model of assortative mating that we have fitted to the data on the Arctic Skua, there is no selection taking place: all matings have the same reproductive success, and no change in gene frequency occurs. However, the assortative mating preferences could produce an advantage of the preferred phenotype in just the same way as O'Donald (1973) postulated. The assortative mating would then be combined with sexual selection. There 
is evidence that this is so in the Arctic Skua population of Fair Isle. In their model with the highest likelihood, O'Donald, Wedd and Davis (1974) estimated that on Fair Isle 18 per cent of the females preferred dark males and 29 per cent preferred intermediate males. Table 4 in this paper shows that 45 per cent of intermediates prefer intermediates according to the model of assortative mating. Intermediates form 60 per cent of the whole population: the 45 per cent of these 60 per cent represents 27 per cent of all females with a preference for intermediates, in close agreement with a value obtained completely independently. Since there is no evidence that dark birds mate assortatively, the preference for dark males must be distributed among all phenotypes of females. According to this theory, sexual selection favours dark males without assortative mating and intermediate males with assortative mating. These possibilities could be tested by observations on the breeding times and reproductive success of the different mating types. Unfortunately, the present data are insufficient for such an analysis.

\section{References}

BURNS, J. M. 1966. Preferential mating versus mimicry: disruptive selection and sex-linked dimorphism in Papilio glaucus. Science, 153, 551-553.

DARwin, C. 1871. The Descent of Man and Selection in Relation to Sex. John Murray, London. KARlin, s. 1969. Equilibrium Behaviour of Population Genetic Models with Non-random Mating. Gordon and Breach, New York.

o'DONALD, P. 1960. Inbreeding as a result of imprinting. Heredity, 15, 79-85.

O'DONALD, P. 1972. Sexual selection for colour phases in the Arctic Skua. Nature, 238, 403-404.

o'DONALD, P. 1973. Frequency-dependent sexual selection as a result of variations in fitness at breeding time. Heredity, 30, 351-368.

o'DONALD, P. 1974. Polymorphisms maintained by sexual selection in monogamous species of birds. Heredity, 32, 1-10.

O'DONALD, P., DAVIS, J. W. F., AND BROAD, R. A, 1974. Variation in assortative mating in two colonies of Arctic Skuas. Nature, 252, 700-701.

O'DONALD, P., WEDD, N. S., AND DAVIS, J. W. F. 1974. Mating preferences and sexual selection in the Arctic Skua. Heredity, 33, 1-16. 